\title{
Shock index thresholds to predict adverse outcomes in maternal hemorrhage and sepsis: A prospective cohort study
}

\author{
Hannah L. Nathan ${ }^{1}$ (D) | Paul T. Seed ${ }^{1}$ | Natasha L. Hezelgrave ${ }^{1}$ | Annemarie De Greeff ${ }^{1}$ | \\ Elodie Lawley $^{1}$ | John Anthony ${ }^{2}$ | Wilhelm Steyn ${ }^{3}$ | David R. Hall ${ }^{3}$ | Lucy C. Chappell ${ }^{1}$ | \\ Andrew H. Shennan ${ }^{1}$
}

${ }^{1}$ Department of Women and Children's Health, King's College London, London, UK

${ }^{2}$ Maternity Department, Groote Schuur Hospital, University of Cape Town, Cape Town, South Africa

${ }^{3}$ Department of Obstetrics and

Gynecology, Stellenbosch University, Cape Town, South Africa

\section{Correspondence}

Hannah L. Nathan, Department of Women and Children's Health, King's College London, 10th floor, North Wing, St Thomas' Hospital, London, SE1 7EH, UK.

Email: hannah.nathan@kcl.ac.uk

\section{Funding information}

Funded by Bill \& Melinda Gates Foundation (Grant ID: OPP1086183).

\begin{abstract}
Introduction: Shock index (SI) is a predictor of hemodynamic compromise in obstetric patients. The SI threshold for action is not well understood. We aimed to evaluate $\mathrm{SI}$ thresholds as predictors of outcomes in obstetric patients.

Material and methods: We undertook a prospective cohort study at three South African hospitals of women with postpartum hemorrhage $(n=283)$ or maternal sepsis $(n=126)$. The "first" and "worst" SI following diagnosis were recorded. SI was compared with conventional vital signs as predictors of outcomes. The performance of $\mathrm{SI}<.9$, SI .9-1.69 and $\mathrm{SI} \geq 1.7$ to predict outcomes (maternal death; Critical Care Unit admission; major procedure; hysterectomy) and hemorrhage-specific outcomes (lowest hemoglobin $<70 \mathrm{~g} /$; ; blood transfusion $\geq 4 \mathrm{IU}$ ) were evaluated.

Results: "First" SI was one of two best performing vital signs for every outcome in postpartum hemorrhage and sepsis. In hemorrhage, risk of all outcomes increased with increasing "first" $\mathrm{SI}$; for blood transfusion $\geq 4$ IU odds ratio was 4.24 (95\% confidence interval 1.25-14.36) for $\mathrm{SI} \geq 1.7 \mathrm{vs} \mathrm{SI}$.9-1.69. In sepsis, risk of all outcomes increased with increasing "worst" SI. Sensitivity, specificity, positive and negative predictive values of "first" SI <.9 vs SI $\geq .9$ for maternal death were $100.0 \%, 55.2 \%, 4.6 \%$ and $100.0 \%$, respectively, in hemorrhage and $80.0 \%, 50.4 \%, 12.3 \%$ and $96.7 \%$, respectively, in sepsis. Conclusions: The shock index was a consistent predictor of outcomes compared with conventional vital signs in postpartum hemorrhage and sepsis. $\mathrm{SI}<.9$ performed well as a rule-out test and $\mathrm{SI} .9-1.69$ and $\mathrm{SI} \geq 1.7$ indicated increased risk of all outcomes in both cohorts. These thresholds may alert to the need for urgent intervention and prevent maternal deaths.
\end{abstract}

\section{KEYWORDS}

blood pressure, heart rate, postpartum hemorrhage, sepsis, shock index, vital signs

\footnotetext{
Abbreviations: AUROC, area under the receiver operating characteristic curve; BP, blood pressure; CCU, Critical Care Unit; HR, heart rate; PPH, postpartum hemorrhage; SI, shock index; VSA, vital signs alert.

This is an open access article under the terms of the Creative Commons Attribution License, which permits use, distribution and reproduction in any medium, provided the original work is properly cited.

(C2019 The Authors. Acta Obstetricia et Gynecologica Scandinavica published by John Wiley \& Sons Ltd on behalf of Nordic Federation of Societies of Obstetrics and Gynecology(NFOG)

[Correction added on 28 June 2019, after first online publication: The copyright line for this article was changed.]
} 


\section{1 | INTRODUCTION}

Approximately 115000 women die from postpartum hemorrhage (PPH), maternal sepsis and abortion complications (often from hemorrhage or sepsis) annually, contributing to almost half of all maternal deaths. ${ }^{1}$ Most deaths are associated with delays in identifying hemodynamic compromise and escalating care ${ }^{2}$ and are avoidable. ${ }^{1,3}$ Early identification of compromise using vital sign measurement (commonly blood pressure [BP] and heart rate [HR]) is critical in preventing maternal mortality and morbidity. In lowand middle-income countries, healthcare providers may not have access to devices measuring vital signs or the training to respond appropriately to abnormal vital signs. It is in these environments that $99 \%$ of maternal deaths occur. ${ }^{4}$ Efforts to improve access to accurate vital sign measurement and training to respond to abnormal vital signs may have a substantial impact on reducing maternal deaths. ${ }^{5}$

The shock index (SI), the ratio of HR to systolic BP, has been shown to be an early predictor of massive transfusion, Intensive Care Unit admission and death in non-obstetric critically ill patients, ${ }^{6}$ trauma ${ }^{7-10}$ and sepsis. ${ }^{11}$ According to retrospective cohorts of women with $\mathrm{PPH}, \mathrm{SI}$ has also been shown to be the most consistent predictor of adverse outcomes compared with individual conventional vital signs, including systolic $\mathrm{BP}^{12,13}$ and studies have proposed an upper limit of normal SI of .9 in PPH. ${ }^{12-14}$ For use as an early warning system, thresholds of $\mathrm{SI}$ have been proposed ( $\mathrm{SI}<.9$, SI .9-1.69 and $\mathrm{SI} \geq 1.7$ ), based on retrospective cohorts of women with $\mathrm{PPH}$ in both wellresourced and low-resourced settings, to indicate increased risk of adverse outcomes. ${ }^{12,13} \mathrm{SI}$ has not been evaluated in maternal sepsis or prospectively evaluated in $\mathrm{PPH}$, and the previously proposed $\mathrm{SI}$ thresholds have not yet been prospectively validated.

This South African facility-level study aimed to evaluate prospectively whether $\mathrm{SI}$ is a consistent predictor of adverse outcomes in both PPH and maternal sepsis and whether the previously determined SI thresholds perform appropriately as predictors of adverse outcomes in both groups of women.

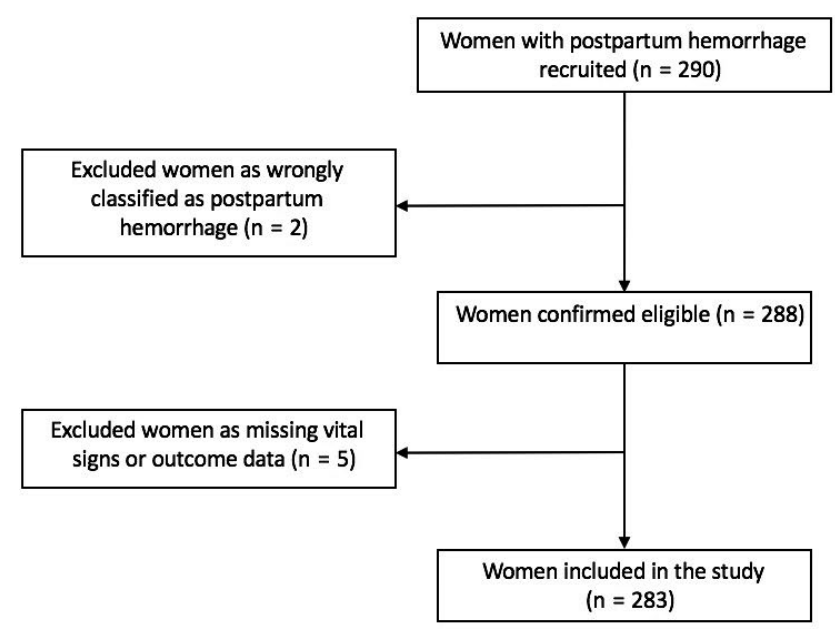

\section{Key message}

Postpartum hemorrhage and sepsis are the leading causes of maternal deaths. The shock index is a useful predictor of adverse outcomes in postpartum hemorrhage and sepsis. Shock index thresholds may alert to the need for urgent intervention and prevent maternal deaths.

\section{2 | MATERIAL AND METHODS}

This prospective cohort study was undertaken between January 2015 and May 2016 at three tertiary maternity units in South Africa (Groote Schuur, Tygerberg and Kimberley Hospitals). Women were eligible if they were diagnosed with either $\mathrm{PPH}$ or maternal sepsis (antepartum or postpartum) during their admission, up until discharge from hospital. PPH was defined as an estimated blood loss $\geq 500 \mathrm{~mL}$ for vaginal deliveries and $\geq 1000 \mathrm{~mL}$ for cesarean deliveries, as defined in studies from similar settings. ${ }^{15}$ Diagnosis of maternal sepsis was based on clinical features determined by the woman's healthcare provider and documented in the patient notes.

Existing BP devices were replaced by the Microlife ${ }^{\circledR}$ CRADLE Vital Signs Alert (VSA), a vital signs device that measures BP and heart rate, is suitable for use in low-resource settings ${ }^{16}$ and is validated as accurate for use in pregnancy, including preeclampsia and low BP in pregnancy. ${ }^{16-18}$ The device incorporates a traffic light early-warning system triggering a green, yellow or red light according to categories of SI: $\mathrm{SI}<.9, \mathrm{SI}$.9-1.69 and $\mathrm{SI} \geq 1.7$, respectively, to alert healthcare providers to abnormalities in vital signs. Anesthetic and recovery areas were allowed access to additional BP devices integrated into existing machines, if requested. Consequently, almost all women had access to the CRADLE VSA and traffic lights triggered by SI. Clinicians used the $H R$ and BP measurements to determine clinical decisions and managed women according to local practice. They were not masked to the traffic light alerts but were not trained to escalate care according to them.

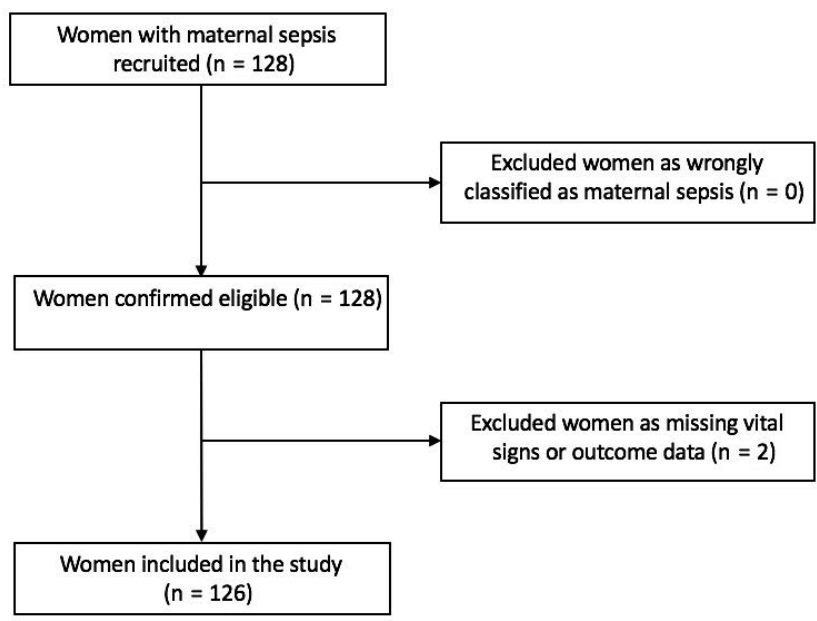

FIGURE 1 Flow diagram of participants 
TAB LE 1 Demographic, admission, delivery, perinatal outcomes and hemorrhage/sepsis details for postpartum hemorrhage and maternal sepsis

\begin{tabular}{|c|c|c|}
\hline Characteristic or outcome & $\begin{array}{l}\text { Postpartum } \\
\text { hemorrhage } \\
(n=283)\end{array}$ & $\begin{array}{l}\text { Maternal sepsis } \\
(\mathrm{n}=126)\end{array}$ \\
\hline \multicolumn{3}{|l|}{ Demographic details } \\
\hline Age at delivery, $y$ & $29.7 \pm 6.2$ & $27.0 \pm 6.6$ \\
\hline Body mass index, $\mathrm{kg} / \mathrm{m}^{2}$ & $30.9 \pm 7.9$ & $31.1 \pm 9.7$ \\
\hline Multiparous & $225,79.5 \%$ & $70,56.0 \%$ \\
\hline \multicolumn{3}{|l|}{ Delivery details } \\
\hline $\begin{array}{l}\text { Gestation at delivery, } \\
\text { weeks }\end{array}$ & $36.3(32.0-39.0)$ & $36.6(32.9-38.9)$ \\
\hline Preterm birth <34 wk & $97,34.3 \%$ & $39,31.5 \%$ \\
\hline Preterm birth <37 wk & $155,54.8 \%$ & $65,52.4 \%$ \\
\hline $\begin{array}{l}\text { Mode of delivery: } \\
\text { Cesarean section }\end{array}$ & $129,45.6 \%$ & $100,80.0 \%$ \\
\hline \multicolumn{3}{|l|}{ Diagnosis details } \\
\hline Estimated blood loss, mL & $\begin{array}{l}1100 \\
(800-1500)\end{array}$ & - \\
\hline $\begin{array}{l}\text { Time from delivery to } \\
\text { diagnosis, minutes }\end{array}$ & $6(0-36)$ & $\begin{array}{l}2586 \\
(1056-4780)^{a}\end{array}$ \\
\hline $\begin{array}{l}\text { Diagnosis made } \\
\text { antenatally }\end{array}$ & NA & $13,10.5 \%$ \\
\hline $\begin{array}{l}\text { Intravenous antibiotics } \\
\text { given }\end{array}$ & NA & $111,88.1 \%$ \\
\hline Blood cultures taken & NA & $114,90.5 \%$ \\
\hline $\begin{array}{l}\text { Blood culture result } \\
\text { positive }\end{array}$ & NA & $35,30.7 \%$ \\
\hline \multicolumn{3}{|l|}{ Perinatal outcomes } \\
\hline Stillbirth & $95,33.6 \%$ & $16,12.7 \%$ \\
\hline Early neonatal death & $8,2.8 \%$ & $3,2.4 \%$ \\
\hline Late neonatal death & $0,0 \%$ & $0,0 \%$ \\
\hline
\end{tabular}

Mean \pm standard deviation, median (interquartile range) and $\mathrm{n}$, percentage are shown.

NA, not applicable.

${ }^{a}$ For postnatal sepsis only.

The "first" and the "worst" sets of BP and HR taken following diagnosis of PPH or sepsis were recorded. The "first" set was defined as those documented immediately after diagnosis and the "worst" set as the set corresponding to the highest SI, documented at any time between diagnosis and discharge (or maternal death). The predefined outcomes included maternal death, maternal Critical Care Unit (CCU) admission, major surgical or invasive procedures and emergency hysterectomy. CCU was defined as a specified Critical Care area providing at least additional monitoring and interventions. ${ }^{19}$ Major surgical or invasive procedures were defined as uterine balloon tamponade, artery ligation/embolization/clamping, hemostatic brace suture, emergency laparotomy for hemorrhage, other procedures related to sepsis or emergency hysterectomy. For hemorrhage, additional outcomes included lowest hemoglobin following diagnosis $<70 \mathrm{~g} / \mathrm{L}$ and blood transfusion $\geq 4$ IU. Perinatal complications were recorded but not treated as outcomes because, by definition, diagnosis of PPH and most sepsis diagnoses were made following delivery. We have previously demonstrated that SI is not influenced by regional anesthesia or mode of delivery, so this data was not collected. ${ }^{20}$

Data were extracted from patient notes reviewed by a local researcher. Data quality checks were undertaken on the database by an external researcher. Discrepancies were adjudicated by an obstetrician. Women with missing vital signs were excluded; women with missing outcomes were included but not analyzed for the outcome for which the data were missing.

\section{1 | Statistical analyses}

Predefined analysis aimed to determine whether SI was selected as a consistent predictor of adverse outcomes. This was calculated using area under the receiver operating characteristic curve (AUROC) values (95\% confidence intervals [CI]) for "first" SI and conventional vital signs (HR, systolic BP, diastolic BP, mean arterial pressure, and pulse pressure) for predicting the predefined outcomes. Mean arterial pressure was defined as ( $2 \times$ diastolic BP + systolic BP)/ 3 and pulse pressure was defined as systolic BP - diastolic BP. Predictor equality of AUROCs across the outcomes was tested using unadjusted chi-square analysis.

The ability of the "first" and "worst" SI categories (SI <.9, SI .9-1.69 and $\mathrm{SI} \geq 1.7$ ) to predict the risk of each outcome was evaluated using post-test probabilities for each category, odds ratios of $\mathrm{SI} .9-1.69$ vs $\mathrm{SI}<.9$ and $\mathrm{SI} \geq 1.7$ vs SI .9-1.69, and non-parametric trend testing of change in risk across the SI categories. ${ }^{21}$ Post-test probability (with 95\% confidence intervals) was defined as the proportion of women with vital signs falling within each category who have the outcome. The $95 \% \mathrm{Cls}$ were included to allow for generalization from the sample to the population with similar characteristics. Post-test probability was used to evaluate the performance of the three categories ( $\mathrm{SI}<.9$, SI .9-1.69, and SI $\geq 1.7$ ), rather than traditional predictive testing using sensitivity, specificity, positive predictive value and negative predictive testing, which is only appropriate when testing one threshold/two categories. ${ }^{22}$ Sensitivity, specificity, and positive predictive value and negative predictive testing were used to evaluate the test performance of the single threshold of SI $<.9$ as a rule-out test at "first" vital signs measurement following diagnosis for the prediction of the two most severe adverse outcomes (emergency hysterectomy and maternal death). Separate analyses were performed for PPH and maternal sepsis. Women with both diagnoses were included in both groups.

A post-hoc power calculation was performed for two principal outcomes, CCU admission and emergency hysterectomy. The rate of CCU admission could be estimated to within $5.9 \%$ and $9.1 \%$ of the true value, for PPH and sepsis, respectively, with $95 \%$ confidence. The rate of emergency hysterectomy could be estimated to within $3.9 \%$ and $6.2 \%$ of the true value, for PPH and sepsis, respectively, with $95 \%$ confidence. Statistical analysis was performed in the statistical package 
TABLE 2 "First" and "worst" shock index values following diagnosis of hemorrhage/sepsis and their timing with respect to diagnosis

\begin{tabular}{|lll|}
\hline SI details & $\begin{array}{l}\text { Postpartum hemorrhage } \\
(\mathbf{n}=283)\end{array}$ & $\begin{array}{c}\text { Maternal sep- } \\
\text { sis ( }=126)\end{array}$ \\
\hline "First" SI after diagnosis & $.95 \pm .32$ & $.99 \pm .82$ \\
\hline "First" HR after diagnosis & $104 \pm 19$ & $114 \pm 19$ \\
\hline "First" systolic BP after diagnosis & $116 \pm 24$ & $126 \pm 22$ \\
\hline Time from diagnosis to "first" SI, minutes & $15(0-44)$ & $0(0-0)$ \\
\hline "First" SI category & & \\
\hline SI <.9 & $153,54.1 \%$ & $60,48.4 \%$ \\
\hline SI .9-1.69 & $116,41.0 \%$ & $63,50.8 \%$ \\
\hline SI $\geq 1.7$ & $14,4.9 \%$ & $1, .8 \%$ \\
\hline Worst" SI after diagnosis & $1.12 \pm .29$ & $1.12 \pm .27$ \\
\hline Worst" HR after diagnosis & $120 \pm 18$ & $130 \pm 20$ \\
\hline Worst" systolic BP after diagnosis & $97 \pm 15$ & $104 \pm 15$ \\
\hline Time from diagnosis to "worst" SI, & $4.23(.42-16.96)$ & 13.8 \\
\hline hours & & $(1.73-46.75)$ \\
\hline "Worst" SI category & & \\
\hline SI <.9 & $60,21.2 \%$ & $24,19.4 \%$ \\
\hline SI .9-1.69 & $206,72.8 \%$ & $94,75.8 \%$ \\
\hline SI $\geq 1.7$ & $17,6.0 \%$ & $6,4.8 \%$ \\
\hline
\end{tabular}

Mean \pm standard deviation or $\mathrm{n}$, percentage or median (interquartile range) are shown. Abbreviations: SI, shock index; HR, heart rate.
STATA (version 11.2). The conventional significance level was set at $P \leq .05$. The study was reported in accordance with STrengthening the Reporting of OBservational studies in Epidemiology (STROBE) guidelines.

\section{Ethical approval}

The study was approved by the Stellenbosch University Ethics Committee (N14/06068, June 2014), University of Cape Town Ethics Committees (410/2014, July 2014) and the University of the Free State Ethics Committee (230408-011, September 2014). Local ethics committees at two of the three sites (Tygerberg Hospital and Kimberley Hospital) required individual informed written consent to be obtained before the woman was enrolled in the study (or waiver of consent was granted if the woman was unconscious). Institutionallevel agreement for the study was given at the third site-Groote Schuur Hospital (ie, individual-level consent was not required).

\section{3 | RESULTS}

A total of 283 women with PPH and 126 women with sepsis were eligible, consented and were included in the analyses (Figure 1). The number of women who declined to take part was not documented. Thirteen women were included in both analyses, having both diagnoses.

Participant characteristics are shown in Table 1. Mean body mass index \pm standard deviation was $30.9 \pm 7.9$ for those with $\mathrm{PPH}$ and $31.1 \pm 9.7$ for those with maternal sepsis. Cesarean section was more common in women with sepsis: 100 women (80\%) with maternal sepsis delivered by cesarean section compared with 129 women (45.6\%) with PPH. Our sites had high cesarean section rates in keeping with referral centers (33\%-55\%), but the high rates in our study population, along with lower gestational age, are in keeping with the adverse obstetric outcomes seen in the study population. In women with sepsis, blood cultures were taken in 114 women (90.5\%); of those, 35 women $(30.7 \%)$ had positive blood culture results.

Vital sign results are shown in Table 2. The mean (standard deviation) "first" and "worst" SI after diagnosis of PPH were $.95 \pm .32$ and $1.12 \pm .29$, respectively; the mean (standard deviation) "first" and "worst" SI after diagnosis of sepsis were $.99 \pm .82$ and $1.12 \pm .27$, respectively. In all, 206 women with PPH (72.8\%) had an SI .9-1.69 as their "worst" SI following diagnosis and 17 women (6.0\%) had an SI $\geq 1.7$ as their "worst" SI following diagnosis.

Table 3 shows the incidence of each outcome. Six women (2.1\%) with PPH and 10 women (7.9\%) with maternal sepsis died while admitted. Of those with PPH, the lowest hemoglobin was $<70 \mathrm{~g} / \mathrm{L}$ in 106 women (37.6\%), and 92 women (32.5\%) received $\geq 4$ IU of blood. A total of 106 women (37.5\%) with $\mathrm{PPH}$ and 61 women (48.4\%) with sepsis were admitted to CCU. Twentyfour women (8.5\%) with PPH and 10 women (7.9\%) with sepsis underwent emergency hysterectomy. Although our blood loss estimates were relatively low, the high rates of transfusion indicate severe hemorrhage and may reflect underestimation of blood loss.

According to AUROC values, SI at "first" vital sign measurement following diagnosis was one of the two best performing vital 
TAB LE 3 Mean \pm standard deviation or $n$, percentage of adverse clinical outcomes for the postpartum hemorrhage group and the maternal sepsis group

\begin{tabular}{|c|c|c|}
\hline Outcomes & $\begin{array}{l}\text { Postpartum } \\
\text { hemorrhage } \\
(n=283 \text { ) }\end{array}$ & $\begin{array}{l}\text { Maternal } \\
\text { sepsis } \\
(n=126)\end{array}$ \\
\hline Maternal death & $6,2.1 \%$ & $10,7.9 \%$ \\
\hline $\begin{array}{l}\text { Lowest hemoglobin following } \\
\text { diagnosis, } g / L\end{array}$ & $77 \pm 19$ & - \\
\hline $\begin{array}{l}\text { Lowest hemoglobin }<70 \mathrm{~g} / \mathrm{L} \text { fol- } \\
\text { lowing diagnosis }\end{array}$ & $106,37.6 \%$ & - \\
\hline Drop in hemoglobin, $g / L(n=247)$ & $27 \pm 21$ & - \\
\hline $\begin{array}{l}\text { Drop in hemoglobin } \geq 2 \mathrm{~g} / \mathrm{L} \\
(\mathrm{n}=247)\end{array}$ & $153,61.9 \%$ & - \\
\hline \multicolumn{3}{|l|}{ Process measures } \\
\hline Blood transfusion & $198,70.0 \%$ & - \\
\hline $\begin{array}{l}\text { Blood transfusion } \geq 4 \mathrm{IU}, \mathrm{n} \text { (\% of } \\
\text { all women) }\end{array}$ & $92,32.5 \%$ & - \\
\hline Number of IU & $3.9 \pm 2.6$ & - \\
\hline Critical Care Unit admission & $106,37.5 \%$ & $61,48.4 \%$ \\
\hline \multicolumn{3}{|l|}{ Procedures } \\
\hline Any & $90,31.8 \%$ & $30,23.8 \%$ \\
\hline $\begin{array}{l}\text { Major surgical or invasive } \\
\text { procedure }\end{array}$ & $66,23.3 \%$ & $55,36.5 \%$ \\
\hline Perineal repair & $31,11.0 \%$ & $3,2.4 \%$ \\
\hline Manual removal of placenta & $14,4.9 \%$ & $1, .8 \%$ \\
\hline Uterine balloon tamponade & $14,4.9 \%$ & $2,1.6 \%$ \\
\hline $\begin{array}{l}\text { Artery ligation/embolization/ } \\
\text { clamping }\end{array}$ & $7,2.5 \%$ & $3,2.4 \%$ \\
\hline Hemostatic brace suturing & $5,1.8 \%$ & $2,1.6 \%$ \\
\hline $\begin{array}{l}\text { Emergency laparotomy for } \\
\text { hemorrhage }\end{array}$ & $15,5.3 \%$ & $1, .8 \%$ \\
\hline $\begin{array}{l}\text { Other procedures related to } \\
\text { sepsis }\end{array}$ & $1, .4 \%$ & $10,7.9 \%$ \\
\hline Emergency hysterectomy & $24,8.5 \%$ & $10,7.9 \%$ \\
\hline
\end{tabular}

IU, international units.

signs for every adverse outcome in both hemorrhage and sepsis groups (Table 4). For example, for predicting risk of emergency hysterectomy, SI gave an AUROC of .79 (95\% Cl; .70-.88) in $\mathrm{PPH}$ and $.73(95 \% \mathrm{Cl} ; .60-.87)$ in maternal sepsis. SI was selected as the most consistent predictor across the outcomes for both groups of women. Tables 5 and 6 show the frequency and percentage of outcomes across the $\mathrm{SI}$ categories, together with the post-test probabilities, odds ratios and the non-parametric trend test, for both groups.

In PPH, statistical testing for trend for every outcome showed a significant increase in risk with higher SI categories at "first" measurement. This was also true for the "worst" SI categories (apart from a nonsignificant trend for maternal death). In sepsis, only one woman had an $\mathrm{SI} \geq 1.7$ at "first" vital signs and this woman had no adverse outcomes, limiting the ability to test for trend in this group.
For "worst" vital signs in women with sepsis, statistical testing for trend for every outcome showed a significant increase in risk with increasing SI categories. The small number of septic women with "worst" SI $\geq 1.7$ were associated with very high risks, with one-third (2 of 6) ending in maternal death. Table 7 shows the sensitivity, specificity, positive predictive value and negative predictive value of "first" SI <.9 in PPH and sepsis. There were no maternal deaths, major procedures or emergency hysterectomies in women with "first" SI <.9.

Sensitivity analysis was performed to determine whether a more stringent categorization of sepsis (the administration of intravenous antibiotics) altered the findings. The additional analysis showed similar odds ratios $(95 \% \mathrm{Cl})$ for all the outcomes for both "first" and "worst" SI categories (data not presented). Therefore, the original definition of sepsis was used to maintain power.

\section{4 | DISCUSSION}

SI was the most consistent predictor, compared with individual conventional vital signs, of all adverse outcomes in women with PPH and maternal sepsis, two of the leading causes of maternal mortality and morbidity.

Previously determined "abnormal" SI categories (SI .9-1.69 and SI $\geq 1.7$ ) were significantly associated with a number of severe adverse outcomes in both cohorts of women. Test performance statistics showed the previously determined upper limit of normal $(\mathrm{SI}<.9)$ to be a good rule-out test for maternal death and emergency hysterectomy in both cohorts of women.

The SI category at "first" vital signs measurement following PPH diagnosis was predictive of all outcomes, including maternal death and emergency hysterectomy, with a stepwise increase in risk from SI <.9 to SI .9-1.69 to SI 21.7. The SI category at "worst" vital signs measurement following maternal sepsis diagnosis was also predictive of all outcomes. As with all vital signs, change over time may be important.

To improve generalizability of the results, multiple centers, limited exclusion criteria and multiple, robust and severe outcomes to assess prediction were used. Sepsis was defined according to clinical features determined by the woman's healthcare provider. Maternal sepsis has been poorly defined in the literature. ${ }^{23}$ Only in 2017, after the completion of this study, did the World Health Organization convene a multidisciplinary international panel of 48 experts to set a definition of maternal sepsis. Maternal sepsis is now defined as organ dysfunction resulting from infection during pregnancy, childbirth, post-abortion or postpartum period. ${ }^{24} \mathrm{We}$ could not determine whether our inclusion criteria correlate with this new definition; however, sensitivity analysis using an alternative categorization of sepsis showed similar findings.

Our objective was to determine whether SI thresholds could aid decision-making for healthcare providers with limited training and resources. Tertiary centers were chosen to ensure sample size and corresponding outcome rates were high enough for 
TA B LE 4 Performance of "first" vital sign parameters to predict adverse clinical outcomes among women with postpartum hemorrhage and maternal sepsis

\begin{tabular}{|c|c|c|c|c|c|c|}
\hline Adverse outcomes & Shock index & Heart rate & Systolic BP & Diastolic BP & Pulse pressure & $\begin{array}{l}\text { Mean arterial } \\
\text { pressure }\end{array}$ \\
\hline \multicolumn{7}{|l|}{ Maternal death } \\
\hline Sepsis & $0.71(0.55-0.86)$ & $0.70(0.54-0.87)$ & $0.53(0.33-0.72)^{a}$ & $0.59(0.33-0.85)$ & $0.52(0.28-0.76)$ & $0.56(0.33-0.78)$ \\
\hline \multicolumn{7}{|l|}{ Critical Care Unit admission } \\
\hline \multicolumn{7}{|l|}{ Hysterectomy } \\
\hline $\mathrm{PPH}$ & $0.79(0.70-0.88)$ & $0.71(0.60-0.82)^{a}$ & $0.73(0.64-0.82)$ & $0.66(0.57-0.75)^{a}$ & $0.63(0.52-0.74)^{a}$ & $0.70(0.61-0.79)^{a}$ \\
\hline Sepsis & $0.73(0.60-0.87)$ & $0.70(0.52-0.87)$ & $0.59(0.45-0.73)$ & $0.59(0.39-0.78)$ & $0.58(0.36-0.81)$ & $0.58(0.36-0.81)$ \\
\hline $\begin{array}{l}\text { Lowest hemoglobin }<70 \mathrm{~g} / \mathrm{L} \\
\text { (PPH) }\end{array}$ & $0.61(0.55-0.68)$ & $0.63(0.56-0.69)$ & $0.54(0.47-0.61)^{\mathrm{a}}$ & $0.51(0.44-0.58)^{a}$ & $0.58(0.51-0.65)$ & $0.52(0.45-0.59)^{a}$ \\
\hline Major procedure (sepsis) & $0.64(0.52-0.75)$ & $0.53(0.40-0.67)^{a}$ & $0.62(0.51-0.73)$ & $0.56(0.44-0.68)$ & $0.63(0.51-0.76)$ & $0.58(0.46-0.69)$ \\
\hline
\end{tabular}

AUROC values given as AUROC $(95 \% \mathrm{CI})$. In bold: highest two AUROC values for each outcome. Results of significance testing for equality of AUROCs using unadjusted chi-square test, with shock index as reference.

${ }^{a}$ Significantly worse than shock index $(P<0.05)$.

TAB LE 5 Frequency and post-test probability of outcomes in women with "first" and "worst" SI <0.9, SI 0.9-1.69, and SI $\geq 1.7$ following hemorrhage diagnosis, odds ratios of SI 0.9-1.69 vs SI <0.9 and SI $\geq 1.7$ vs SI 0.9-1.69 and non-parametric trend test for worsening SI category (SI $<0.9$ to $\mathrm{SI} 0.9-1.69$ to $\mathrm{SI} \geq 1.7$ )

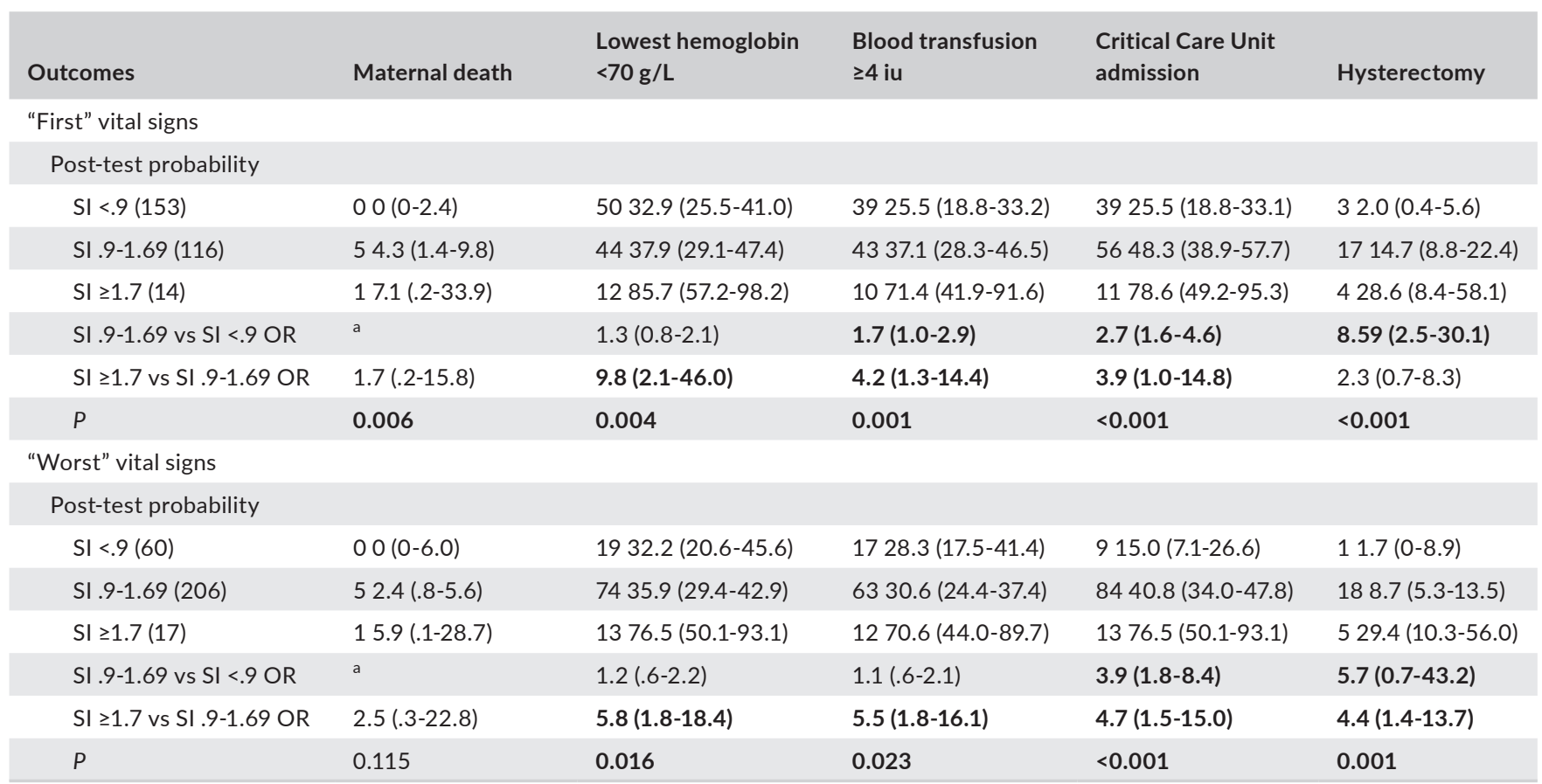

Post-test probability values are given as n, \% (95\% confidence interval). Odds ratios given as OR ( $95 \%$ confidence intervals). Values in bold indicate statistical significance. All $P$-values are based on the non-parametric test for trend.

${ }^{\mathrm{a}}$ Not calculable due to $0 \%$.

meaningful analysis. It would have been considerably more challenging to undertake such a study in community low- and middle-income countries, where the device is likely to show greatest benefit; outcome rates would have been fewer and a much larger sample size would have been required. Although the number of women with $\mathrm{SI} \geq 1.7$ was relatively small, severe adverse outcomes were common, unique in comparison with similar maternal health studies. 
TAB LE 6 Frequency and post-test probability of outcomes in women with "first" and "worst" SI <.9, SI .9-1.69, and SI $\geq 1.7$ following sepsis diagnosis, odds ratios of SI .9-1.69 vs SI <.9 and SI $\geq 1.7$ vs SI .9-1.69 and non-parametric trend test for worsening SI category (SI <.9 to SI 9-1.69 to $\mathrm{SI} \geq 1.7)$

\begin{tabular}{|c|c|c|c|c|}
\hline Outcomes & Maternal death & $\begin{array}{l}\text { Critical Care Unit } \\
\text { admission }\end{array}$ & Major procedure & Hysterectomy \\
\hline \multicolumn{5}{|l|}{ "First" vital signs } \\
\hline \multicolumn{5}{|l|}{ Post-test probability } \\
\hline $\mathrm{SI}<0.9(60)$ & $23.3(0.4-11.5)$ & $2338.3(26.1-51.8)$ & $915.0(7.1-26.6)$ & $23.3(0.4-11.5)$ \\
\hline SI 0.9-1.69 (64) & $812.5(5.6-23.2)$ & $3757.8(44.8-70.1)$ & $1625.0(15.0-37.4)$ & $812.5(5.6-23.2)$ \\
\hline $\mathrm{SI} \geq 1.7(1)$ & $0^{a}$ & $0^{a}$ & $0^{a}$ & $0^{a}$ \\
\hline SI 0.9-1.69 vs $\mathrm{SI}<.9$ OR & $4.14(0.84-20.36)$ & $2.20(1.07-4.52)$ & $1.89(0.76-4.68)$ & $4.14(0.84-20.36)$ \\
\hline $\mathrm{SI} \geq 1.7$ vs $\mathrm{SI} .9-1.69 \mathrm{OR}$ & a & a & a & a \\
\hline$P$ & 0.083 & 0.065 & 0.226 & 0.083 \\
\hline \multicolumn{5}{|l|}{ "Worst" vital signs } \\
\hline \multicolumn{5}{|l|}{ Post-test probability } \\
\hline $\mathrm{SI}<.9(24)$ & $0^{\mathrm{a}}$ & $729.2(12.6-51.1)$ & $0^{a}$ & $0^{\mathrm{a}}$ \\
\hline SI .9-1.69 (95) & $88.4(3.7-15.9)$ & $4951.6(41.1-62.0)$ & $2324.2(16.0-34.1)$ & $88.4(3.7-15.9)$ \\
\hline $\mathrm{SI} \geq 1.7(6)$ & $233.3(4.3-77.8)$ & $466.7(22.2-95.7)$ & $233.3(4.3-77.8)$ & $233.3(4.3-77.8)$ \\
\hline SI .9-1.69 vs $\mathrm{SI}<.9$ OR & a & $2.59(0.98-6.81)$ & a & a \\
\hline $\mathrm{SI} \geq 1.7$ vs $\mathrm{SI} .9-1.69 \mathrm{OR}$ & $5.44(0.86-34.42)$ & $1.88(0.33-1.74)$ & $1.57(0.27-9.11)$ & $5.44(0.86-34.42)$ \\
\hline$P$ & 0.016 & 0.032 & 0.008 & 0.016 \\
\hline
\end{tabular}

Post-test probability values are given as $\mathrm{n}, \%$ ( $95 \%$ confidence interval), odds ratios given as OR ( $95 \%$ confidence intervals), values in bold indicate statistical significance. All P-values are based on the non-parametric test for trend. Major procedures included uterine balloon tamponade, artery ligation/embolization/clamping, hemostatic brace suture, emergency laparotomy for hemorrhage, other procedure related to sepsis, emergency hysterectomy.

${ }^{\mathrm{a}}$ Not calculable due to $0 \%$.

TAB LE 7 Test performance statistics for "first" SI <.9 in prediction of adverse outcomes in women with postpartum hemorrhage or maternal sepsis

\begin{tabular}{|c|c|c|c|c|}
\hline & PPH & Sepsis & $\mathrm{PPH}$ & Sepsis \\
\hline Outcomes & Maternal death total $\mathrm{N}=6$ & Maternal death $\mathrm{N}=10$ & Hysterectomy N = 24 & Hysterectomy $\mathrm{N}=10$ \\
\hline Sensitivity (\%) n/N & $100.0(54.1-100.0) 6 / 6$ & $80.0(44.4-97.5) 8 / 10$ & 87.5 (67.6-97.3) 21/24 & $80.0(44.4-97.5) 8 / 10$ \\
\hline Specificity (\%) n/N & $55.2(49.2-61.2) 153 / 277$ & $50.4(41.0-59.9)$ 58/115 & $\begin{array}{l}57.9(1.69-2.56) \\
150 / 259\end{array}$ & $\begin{array}{l}50.4(41.0-59.9) \\
58 / 115\end{array}$ \\
\hline PPV (\%) n/N & $4.6(1.7-9.8) 6 / 130$ & $12.3(5.5-22.8) 8 / 65$ & $16.2(10.3-23.6) 21 / 130$ & $12.3(5.5-22.8) 8 / 65$ \\
\hline NPV (\%) n/N & $\begin{array}{l}100.0(97.6-100.0) \\
153 / 153\end{array}$ & 96.7 (88.5-99.6) 58/60 & $\begin{array}{l}98.0(94.4-99.6) \\
150 / 153\end{array}$ & $\begin{array}{l}96.7(88.5-99.6) \\
58 / 60\end{array}$ \\
\hline
\end{tabular}

PPH, postpartum hemorrhage; PPV, positive predictive value; NPV, negative predictive value.

The traffic light early-warning system and BP and HR values were visible to healthcare providers (as it was not possible to mask healthcare professionals to the lights and not ethical to withhold the absolute numbers). Healthcare providers could escalate care in response to the traffic lights that were triggered according to SI categories (although SI values were not displayed on the device); however, healthcare providers received no specific training on escalation of care according to the traffic lights. Therefore, outcomes may have improved in response to BP and HR, and to a lesser extent, in response to the traffic lights. As we have found significant associations between the SI categories and adverse outcomes, not only do our results remain valid, but arguably the association would likely have been even stronger if clinicians were masked to the traffic lights.

Our study reinforces the role of $\mathrm{SI}$ as a consistent marker of compromise in obstetrics. A 2013 systematic review assessing the association between vital signs and blood loss identified three obstetric studies involving SI. ${ }^{25}$ All three studies included only women in early pregnancy and only one was deemed of high methodological quality. ${ }^{26}$ In that study, SI performed better than all individual conventional vital signs for predicting ruptured ectopic pregnancy, with an AUROC of $.84\left(95 \% \mathrm{Cl}\right.$; .78-.88). ${ }^{26}$ Since the systematic 
review, two other retrospective obstetric studies have suggested SI to be a reliable marker of deterioration. However, both studies included only women with PPH, with limited statistical analysis and fewer, less meaningful clinical outcomes. ${ }^{14,27}$

Our research team previously performed two retrospective prediction studies to evaluate SI and conventional vital signs as predictors of adverse outcomes in women with PPH (high- and low-income settings) and to develop potential traffic light early-warning system thresholds for the CRADLE VSA. ${ }^{12,13}$ The utility of SI in maternal sepsis has not previously been explored. This prospective study has confirmed that $\mathrm{SI}$ was one of the two best performing vital signs across the outcomes in PPH and maternal sepsis. The study also validated the previously determined SI thresholds in women with PPH and sepsis. The pathophysiology and clinical courses of these conditions differ and SI varies from minute to minute in each woman. Despite this, and in this study, one-off SI values consistently predicted a variety of severe adverse outcomes in both conditions.

The previously proposed upper limit of normal (SI <.9, green traffic light) performed well as a rule-out test, according to predictive analysis statistics. SI .9-1.69 and SI $\geq 1.7$ corresponded to stepwise increased risk of multiple outcomes in both cohorts of women. For PPH, the "first" SI following diagnosis was most useful, although both "first" and "worst" had predictive value. For maternal sepsis, the "worst" rather than the "first" SI following diagnosis was most useful. These findings reflect the clinical course of each condition, with complications of hemorrhage evolving more quickly than in sepsis. Complications of sepsis tend to evolve more insidiously and repeated measurements may be more useful in sepsis.

The need for an obstetric early warning system is not only relevant for low- and middle-income countries. The latest UK Confidential Enquiries into Maternal Deaths and Morbidity highlights that avoidable maternal deaths and near-misses are often related to delays in recognition of hemodynamic compromise. ${ }^{28}$ Modified early obstetric warning system charts are recommended by the report and are now commonplace internationally. Despite this, a standardized modified early obstetric warning system does not exist and the evidence for their thresholds is limited. ${ }^{29}$ They are complicated to use and inappropriate for low-resource settings, where temperature and oxygen saturation is often not routinely measured.

\section{5 | CONCLUSION}

SI thresholds evaluated in this study may aid decision-making by healthcare providers caring for women with PPH or maternal sepsis in low-resource settings, allowing for earlier interventions for those at highest risk of adverse outcomes. These thresholds have been incorporated into the CRADLE VSA traffic light early-warning system, together with conventional hypertension thresholds for women with hypertension disorders of pregnancy. A similar study of the performance of the early-warning system in preeclampsia was also undertaken. ${ }^{30}$ By assisting in the prediction for all three conditions, the CRADLE VSA could identify women at greatest risk of the three leading causes of maternal mortality and morbidity worldwide. To confirm the value of SI there is a need for more data from low-income settings where adverse outcomes are more prevalent.

Although recognition of the need for intervention is but one aspect of a functioning health system, it is the gateway to that system. We are undertaking a stepped-wedge randomized control trial to assess whether a maternal mortality and morbidity are reduced in 10 low-income sites, introducing the CRADLE VSA and a simple education package at community-, clinic- and hospital-levels.

\section{ACKNOWLEDGMENTS}

Thank you to Erika van Papendorp and Pippa Readhead, research assistants, for collecting the data locally.

\section{CONFLICT OF INTEREST}

The authors have stated explicitly that there are no conflicts of interest in connection with this article.

\section{ORCID}

Hannah L. Nathan (iD https://orcid.org/0000-0002-4708-8973

\section{REFERENCES}

1. Say L, Chou D, Gemmill A, et al. Global causes of maternal death: a WHO systematic analysis. Lancet Glob Health. 2014;2(6):e323 -e333.

2. Cantwell R, Clutton-Brock T, Cooper G, et al. Saving Mothers' Lives: reviewing maternal deaths to make motherhood safer: 2006-2008. The Eighth Report of the Confidential Enquiries into Maternal Deaths in the United Kingdom. BJOG. 2011;118(Suppl 1):1-203.

3. Grimes DA, Benson J, Singh S, et al. Unsafe abortion: the preventable pandemic. Lancet. 2006;368(9550):1908-1919.

4. Kassebaum NJ, Barber RM, Bhutta ZA, et al. Global, regional, and national levels of maternal mortality, 1990-2015. Lancet. 2016;388:1775-1812.

5. World Health Organization. The World Health Report 2005: Make Every Mother and Child Count. Geneva: WHO; 2005.

6. Rady MY, Smithline HA, Blake H, Nowak R, Rivers E. A comparison of the shock index and conventional vital signs to identify acute, critical illness in the emergency department. Ann Emerg Med. 1994;24(4):685-690.

7. McNab A, Burns B, Bhullar I, Chesire D, Kerwin A. A prehospital shock index for trauma correlates with measures of hospital resource use and mortality. Surgery. 2012;152(3):473-476.

8. Vandromme MJ, Griffin RL, Kerby JD, McGwin G Jr, Rue LW 3rd, Weinberg JA. Identifying risk for massive transfusion in the relatively normotensive patient: utility of the prehospital shock index. J Trauma. 2011;70(2):384-388

9. Cannon CM, Braxton CC, Kling-Smith M, Mahnken JD, Carlton E, Moncure M. Utility of the shock index in predicting mortality in traumatically injured patients. J Trauma. 2009;67(6):1426-1430.

10. Sloan EP, Koenigsberg M, Clark JM, Weir WB, Philbin N. Shock index and prediction of traumatic hemorrhagic shock 28-day mortality: data from the DCLHb resuscitation clinical trials. West J Emerg Med. 2014;15(7):795. 
11. Asaari $\mathrm{H}$. Value of shock index in prognosticating the short term outcome of death for patients presenting with severe sepsis and septic shock in the emergency department. Med J Malaysia. 2012;67(4):407.

12. Nathan HL, El Ayadi AM, Hezelgrave NL, et al. Shock index: an effective predictor of outcome in postpartum haemorrhage? BJOG. 2015;122(2):268-275.

13. El Ayadi AM, Nathan HL, Seed PT, et al. Vital sign prediction of adverse maternal outcomes in women with hypovolemic shock: the role of shock index. PLoS ONE. 2016;11(2):e0148729.

14. Le Bas A, Chandraharan E, Addei A, Arulkumaran S. Use of the "obstetric shock index" as an adjunct in identifying significant blood loss in patients with massive postpartum hemorrhage. Int J Gynaecol Obstet. 2013;124:253-255.

15. WOMAN Trial Collaborators. Effect of early tranexamic acid administration on mortality, hysterectomy, and other morbidities in women with post-partum haemorrhage (WOMAN): an international, randomised, double-blind, placebo-controlled trial. Lancet. 2017;389(10084):2105-2116.

16. de Greeff A, Nathan H, Stafford N, Liu B, Shennan AH. Development of an accurate oscillometric blood pressure device for low resource settings. Blood Press Monit. 2008;13(6):342-348.

17. Nathan HL, de Greeff A, Hezelgrave NL, Chappell LC, Shennan $\mathrm{AH}$. An accurate semiautomated oscillometric blood pressure device for use in pregnancy (including pre-eclampsia) in a low-income and middle-income country population: the Microlife 3AS1-2. Blood Press Monit. 2015;20(1):52-55.

18. Nathan HL, de Greeff A, Hezelgrave NL, Chappell LC, Shennan AH. Accuracy validation of the Microlife 3AS1-2 blood pressure device in a pregnant population with low blood pressure. Blood Press Monit. 2015;20(5):299-302.

19. Intensive Care Society Report. Levels of Critical Care for Adult Patients. London: Intensive Care Society; 2009.

20. Nathan HL, Cottam K, Hezelgrave NL, et al. Determination of normal ranges of shock index and other haemodynamic variables in the immediate postpartum period: a cohort study. PLOS ONE. 2016;11(12):e0168535.

21. Conover WJ. Nonparametric Trend Test. Practical Nonparametric Statistics, 3rd edn. New York: Wiley; 1999:169-175.

22. Straus SE, Glasziou PP, Richardson WS, Haynes RB. Diagnosis and screening. In: Horne T, ed. Evidence-Based Medicine: How to Practice and Teach It, 4th edn. London: Churchill Livingstone; 2011:137-167.

23. Bonet M, Pileggi VN, Rijken MJ, et al. Towards a consensus definition of maternal sepsis: results of a systematic review and expert consultation. Reprod Health. 2017;14(1):67.

24. World Health Organization. Statement on Maternal Sepsis. Geneva: WHO; 2017.

25. Pacagnella RC, Souza JP, Durocher J, et al. A systematic review of the relationship between blood loss and clinical signs. PLoS ONE. 2013;8(3):e57594.

26. Birkhahn RH, Gaeta TJ, Bei R, Bove JJ. Shock index in the first trimester of pregnancy and its relationship to ruptured ectopic pregnancy. Acad Emerg Med. 2002;9(2):115-119.

27. Sohn $\mathrm{CH}$, Kim WY, Kim SR, et al. An increase in initial shock index is associated with the requirement of massive transfusion in emergency department patients with primary postpartum hemorrhage. Shock. 2013;40(2):101-105.

28. Knight M, Tuffnell D, Kenyon S, Shakespeare J, Brocklehurst P, Kurinczuk JJ (eds); on behalf of MBRRACE-UK. Saving Lives, Improving Mothers' Care-Surveillance of maternal deaths in the UK 2012-14 and lessons learned to inform maternity care from the UK and Ireland Confidential Enquiries into Maternal Deaths and Morbidity 2009-14. Oxford: National Perinatal Epidemiology Unit, University of Oxford; 2016.

29. Singh S, McGlennan A, England A, Simons R. A validation study of the CEMACH recommended modified early obstetric warning system (MEOWS). Anaesthesia. 2012;67(1):12-18

30. Nathan HL, Seed PT, Hezelgrave NL, et al. Early warning system hypertension thresholds to predict adverse outcomes in pre-eclampsia: a prospective cohort study. Pregnancy Hypertens. 2018;12:183-188.

How to cite this article: Nathan HL, Seed PT, Hezelgrave NL, et al. Shock index thresholds to predict adverse outcomes in maternal hemorrhage and sepsis: A prospective cohort study. Acta Obstet Gynecol Scand. 2019;98:1178-1186. https://doi. org/10.1111/aogs.13626 